\section{PROTEINS}

\section{Mercurial Enzyme}

from our Molecular Biology Correspondent

AMONG the many recent examples of survival of enzymes in working condition in face of the most extreme forms of chemical violence and abuse, none is more astonishing than that just described by Sperling and Steinberg (J. Biol. Chem., 246, 715; 1971). Earlier work from the same laboratory had shown that considerable liberties could be taken with the disulphide bonds of pancreatic ribonuclease without complete annihilation of activity. On the one hand, two of the four bonds could be reduced, leaving an active product and, on the other, one disulphide bond could be reduced and a mercury atom inserted, also with no untoward consequences.

Sperling and Steinberg now describe the insertion of mercury into all four bonds. Mercuric ions will react with thiols to form the group $-\mathrm{S}-\mathrm{Hg}-\mathrm{S}$-, this being $3 \AA$ longer than -S-S-. When mercuric chloride is added to reduced ribonuclease in dilute solution a product, homogeneous by all criteria and sedimenting at the rate associated with the native enzyme, is generated, which contains four atoms of mercury per molecule of protein. This material has 25 per cent activity towards cyclic CMP and 5 per cent towards RNA. Sperling and Steinberg have gone to all necessary lengths to demonstrate that the activity is a property of the modified ribonuclease and is not associated with a contaminating fraction of reoxidized enzyme. In the first place the material migrates as a single zone in gel electrophoresis, but with a mobility different from that of native ribonuclease.

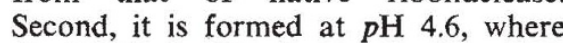
disulphide bonds do not re-form and reduced enzymes do not renature. Third, the modified ribonuclease, unlike the native, is digested by trypsin at a moderately rapid rate (though more slowly than the unfolded oxidized protein); the loss of enzymatic activity parallels the rate of digestion, and cannot therefore be due to a contaminant of the resistant native enzyme.

In addition to the proteolytic lability, the mercury complex is substantially devoid of the Cotton effect seen in circular dichroism in the region of tyrosine absorption. The environment of one tyrosine is therefore appreciably different, which is consistent with the further observation that one of the three untitratable tyrosines of the native enzyme is normalized in the modified form. Not only then is the mercury sufficient to induce correct refolding in spite of the $3 \AA$ stretch that it causes, but the structure can also absorb this very large distortion with little enough discomfort that activity is preserved.

A key to this phenomenon may perhaps be found in the analysis by Lee and Richards (J. Mol. Biol., 55, 379; 1971) of side chain packing in known globular protein structures. In ribonuclease they find that there are three cavities, one of them of considerable volume (calculated as $0.375 \AA$ ). This may be important in absorbing some of the slack generated by intruding elements, such as the four mercury atoms.

From an artificial to a natural change of geometry-Bustin and ConwayJacobs (J. Biol. Chem., 246, 615; 1971) have re-examined the activation process in pepsinogen. As with other zymogens activation is triggered by a specific proteolytic cleavage of the chain. This much was established more than thirty years ago, but more recently evidence of more complex interrelations has accrued, for the nature of the reaction depends on the $p \mathrm{H}$. In order to follow the appearance of proteolytic activity pepsinogen was added at acid $p \mathrm{H}$ to a protein substrate, haemoglobin, and the rate of digestion followed. When partly predigested haemoglobin was used proteolysis

\title{
Cooperating Cancer Viruses
}

IT has been suggested that it might be possible to screen for individuals and families running an abnormally high risk from cancer by measuring the susceptibility of human cells to transformation by the DNA tumour virus SV40. The idea stems from the discovery that cells taken from patients with such diseases as Down's syndrome, Klinefelter's syndrome and Franconi's anaemia - diseases associated with a high incidence of cancer-and from a family with a history of multiple sarcomas, are all markedly more susceptible to transformation by SV40 than the cells of normal people. In next week's Nature New Biology, Rhim and his colleagues report experiments which may have some bearing on these observations. They have found that rat embryo cells infected with the Rauscher strain of mouse leukaemia virus are more efficiently transformed by SV40 than comparable uninfected rat embryo cells. It is not inconceivable that these rat cells infected by murine leukaemia virus and human cells from people who run a high risk of developing cancer have certain properties in common which render them unusually susceptible to transformation by SV40.

The established lines of rat embryo cells infected by the Rauscher leukaemia virus, which Rhim et al. selected, had been producing progeny virus particles for more than 18 months whereas parallel lines of uninfected cells neither contained began without a lag, and the specific activity was independent of pepsinogen concentration, and was not increased by added pepsin. This strongly suggests that the pepsinogen is self-activating under these conditions. This inference was supported by the elegant conceit of binding the pepsinogen covalently to a 'Sepharose' matrix, so that all the molecules are isolated from each other, and showing that at $\mathrm{pH} 2$ the zymogen in this state underwent rapid activation. In these circumstances it is scarcely possible that traces of pepsin are involved, or that the reaction is intermolecular. The internal cleavage to expose a second $\mathrm{N}$-terminal group could be followed in the matrix-bound protein. At acid $p \mathrm{H}$ the conformation evidently changes in such a manner as to create an active site, which forthwith bites another part of its own chain. Whether the haemoglobin substrate is digested by pepsinogen or pepsin is not revealed. This is the second example of a self-activating zymogen, the other being that of streptococcal protease, described last year by Bustin, Lim, Stein and Moore. leukaemia virus antigens nor produced the virus itself. Cultures of the two classes of cells were exposed to SV40 at the same multiplicities of infection, subcultured after a week and then allowed to grow for a further two weeks. By that time foci of transformed cells could easily be distinguished, because within the foci the cells grew to several layers deep. There were up to about ten-fold more foci of transformed cells in cultures of the cells infected with leukaemia virus than in the corresponding uninfected cultures. Moreover, the titres of SV40 induced tumour antigen were also much higher in the former than in the latter cultures.

Clearly, rat embryo cells infected with murine leukaemia virus are more susceptible to transformation by the DNA tumour virus SV40 than comparable cells which are not infected by the leukaemia agent. This is the first example of infection by an RNA tumour virus enhancing a cell's susceptibility to transformation by a DNA virus and the molecular basis of the phenomenon has yet to be deciphered. For example, it might be that the cells infected with the leukaemia virus more efficiently take up or uncoat SV40, or perhaps there are other intracellular changes in these cells which promote some later step in transformation by SV40. It will, however, be of great interest, and possibly of relevance to human cancer, if the former possibility proves to be the case. 